\title{
Plasticity in primary somatosensory cortex resulting from environmentally enriched stimulation and sensory discrimination training
}

\author{
ELIANA GUIC ${ }^{1}$, XIMENA CARRASCO ${ }^{2}$, EUGENIO RODRÍGUEZ $^{1}$, \\ IGNACIO ROBLES ${ }^{3}$ and MICHAEL M. MERZENICH ${ }^{4}$
}

\footnotetext{
${ }^{1}$ Department of Psychology, Faculty of Social Sciences, Pontificia Universidad Católica de Chile

${ }^{2}$ Department of Pediatrics, School of Medicine, Universidad de Chile

${ }^{3}$ Department of Surgery Hospital del Salvador, School of Medicine, Universidad de Chile (Resident)

${ }^{4}$ Keck Center for Integrative Neurosciences, UCSF, San Francisco, CA 94143, U.S.A.
}

\begin{abstract}
We studied primary-somatosensory cortical plasticity due to selective stimulation of the sensory periphery by two procedures of active exploration in adult rats. Subjects, left with only three adjacent whiskers, were trained in a roughness discrimination task or maintained in a tactile enriched environment. Either training or enrichment produced 3-fold increases in the barrel cortex areas of behaviorally-engaged whisker

representations, in their zones of overlap. While the overall areas of representation expanded dramatically, the domains of exclusive principal whisker responses were virtually identical in enriched vs normal rats and were significantly smaller than either group in roughness discrimination-trained rats. When animals were trained or exposed to enriched environments with the three whiskers arrayed in an arc or row, very equivalent overlaps in representations were recorded across their greatly-enlarged whisker representation zones. This equivalence in distortion in these behavioral preparations is in contradistinction to the normal rat, where overlap is strongly biased only along rows, probably reflecting the establishment of different relations with the neighboring cortical columns. Overall, plasticity phenomena are argued to be consistent with the predictions of competitive Hebbian network plasticity.
\end{abstract}

Key terms: cortical plasticity, roughness discrimination, environmental enrichment, barrel cortex, active exploration

\section{INTRODUCTION}

The general purpose of the present work was to study the functional reorganization of the primary somatosensory cortex (SI) generated by a sensory- perceptual learning task contrasted with SI plasticity driven by environmental sensory enrichment.

Neuroscience is now sharply focused on the determination of plastic events in the neocortex that underlie learning and memory. Currently, evidence points to an important relevant role of primary cortices as memory loci, in contradistinction to the more traditional views that behavioral-driven changes may arise in association cortices.
Given its importance, the unraveling of primary cortical phenomenology and mechanisms involved in consolidation processes for learning and memory become crucial.

There has traditionally been a consensus about the essential stability of primary cortical maps representing the sensory periphery in adult subjects, in contrast to the great plasticity that characterized the developing nervous system. That conclusion has been challenged by a large number of studies showing that primary somatosensory cortical representations can be substantially remodeled following alterations of sensory inputs in adult

Correspondence should be addressed to: Eliana Guic, eguic@uc.cl, (56-2) 35448 45, Departamento de Psicología. Pontificia Universidad Católica de Chile. Avenida Vicuña Mackenna 4860. Santiago. 
animals (Kalaska \& Pomeranz, 1979; Rasmusson, 1982; Merzenich et al., 1983a, Merzenich et al., 1983b, Merzenich et al., 1984; Wall \& Cusick, 1984; Rasmusson \& Dykes, 1988; Kaas et al., 1990; Calford \& Tweedale, 1991; Kaas, 1991; Pons et al., 1988). In those studies, amputation, denervation or central nervous system lesions altering sensory system input sources resulted in a re-mapping of the SI cortical sectors formerly representing the damaged or lost input. In general, changes resulted in an increased responsiveness and elaborated representation of the deafferented cortical area to the stimulation of sensory inputs whose representations neighbored the input loss.

Studies of Jenkins et al. (1990), and later, of Rencazone et al. (1992) used selective stimulation to demonstrate that sensory experience was also capable of producing large-scale cortical reorganization. Their findings constituted important evidence for the relationship between cortical plasticity and nondeclarative memory. In the Jenkins study, a large-scale change in the cortical representation of the hand in SI in the monkey was induced by selective use of a limited sector of the periphery, in an operant behavior. Their results implied that competitive functional reorganization of the cortex underlies changes in the functional capacities of the intact primary cortex. Usedependent plasticity has subsequently been studied in SI in a number of related models that collectively indicate that plasticity processes in perceptual and skill learning account for the acquisition and retention (memory) of learned abilities in adults .

Several works have contributed to the discussion as to whether mechanisms underlying functional cortical reorganization are of cortical origin. Some studies have demonstrated that SI plasticity, produced by a relevant sensory stimulation, is not accompanied by changes in subcortical receptive fields (e.g. Wallace \& Fox, 1999). However, in experiments in which a rather extensive lesion of the nervous system was performed by deafferentation, large subcortical changes were recorded in the thalamus and brain stem, which must contribute to the changes expressed in SI, but with changes almost certainly also contributed to by intracortical mechanisms (Krupa et al., 1999; Parker \& Dostrovsky, 1999; Xu \& Wall, 1999). Overall, evidence points to a cortical origin for use-dependent reorganization - which is our current interest - but also, to a combination of cortical and subcortical reorganization contributing to the long-term representational remodeling following a peripheral loss or lesion.

In the present study, we used the vibrissal somatosensory system of the rat to further investigate these issues. Several recent publications have stressed the importance of this system in studying behavioral and neurophysiological mechanisms underlying memory and perception (Petersen, 2007; Brecht, 2007). Our specific interest lies in the effects of the engagement of vibrissae in a procedural learning task on cortical representation, as compared to the effects of sensory enrichment.

To study the effect of selective vibrissal stimulation, other authors have left one or two intact vibrissae, thereby depriving the rest, which led to an increase in cortical representation (Glazewski \& Fox, 1996) or to an increase in stimulus evoked response magnitude (Armstrong-James et al., 1994). In those studies, rats were returned to normal housing conditions, where they differentially stimulated intact vibrissae for relatively short periods of time. After long lasting stimulation in this environment (28 30 days), relatively modest changes in cortical activity were recorded (ArmstrongJames et al., 1994). In a later study, Polley et al. (2003) actually showed a decrease in functional representation documented by optical imaging, after a brief daily period of stimulation of a single vibrissa in an enriched environment. We believe that this absence of a change or decrease in cortical response may be attributed to habituation, which could be expected to result from a lack of active exploration of relevant tactile stimuli. The stimulation condition must provide important and rewarding stimuli that are relevant enough to generate active exploration and stimulation (Weinberger, 1995; Merzenich et al., 1995, 2001). Here 
we used sensory stimulation in a tactually enriched environment that was constantly changing in terms of stimuli characteristics and food location. This provided a stimulation condition that avoids habituation and enables a wide range of self-generated tactile experiences. No earlier published studies have compared the effect of stimulation and learning on cortical functional reorganization. We believe that this issue is of importance to the search for the mnemonic trace characteristics in the neocortex.

In normal rats, the extent of overlapping representational areas varies systematically across the barrel field, but intra-row overlapping area bias is always predominant in a constant proportion (Guic et al., 2008). In this companion paper we described in normal intact rats, that the cortical representation of a vibrissa was significantly more overlapped with the cortical representation of other vibrissae located in its same row than with CRs of vibrissae located in its same arc, in effect, overlapping showed a directional bias toward the rows.

We were therefore interested in looking for the effect of selective stimulation of 3 vibrissae in an arc (B1C1D1) or in a row $(\mathrm{C} 1 \mathrm{C} 2 \mathrm{C} 3)$ in an enriched tactile environment or in a roughness discrimination training task.

The main objectives of the present study were:

1. To compare the effects of active exploration in an enriched tactile environment or in a vibrissal learning situation, looking for effects on exclusive and overlapping cortical representations of selectively stimulated vibrissae.
2. To compare CR of row vs arc pattern of selective sensory stimulation.

METHOD

Subjects. Twelve experimentally naive male Sprague-Dawley rats were used. Rats were approximately 45 days of age at the beginning of the study. Subjects were randomly assigned to one of the four groups. All procedures involving the animals were made in accordance with the local Committee of Bioethics and the NIH Guidelines for the Care and Use of Laboratory Animals.

Experimental Design. Table I shows the experimental design. Four rats were stimulated in an enriched environment and four were trained. In each condition two rats had three vibrissae stimulated or trained in a row or an arc, while the rest were deprived. Electrophysiological responses from these rats were compared to those of normal control rats. Data from these normal rats, with all their vibrissae intact and maintained in a standard animal colony environment, are further analyzed in detail in a companion publication (Guic et al., 2008).

\section{PROCEDURES}

Selective Stimulation. On day 1 , each rat had all its vibrissae cut short, except for 3 on the left side, ordered in a row $(\mathrm{C} 1, \mathrm{C} 2$ and C3) or in an arc (B1, C1 and D1). Deprived vibrissae, which approximately grow $1 \mathrm{~mm}$ in 24 hours, were trimmed daily. Rats were

TABLE I

\begin{tabular}{|c|c|c|c|c|c|}
\hline & \multicolumn{2}{|c|}{$\begin{array}{l}\text { Trained group } \\
\text { (4 rats) }\end{array}$} & \multicolumn{2}{|c|}{$\begin{array}{l}\text { Enriched group } \\
\text { (4 rats) }\end{array}$} & \multirow{2}{*}{$\begin{array}{c}\text { Control group } \\
\text { (4 rats) }\end{array}$} \\
\hline & $\begin{array}{l}\text { Trained } \\
\text { vibrissae }\end{array}$ & $\begin{array}{l}\text { Deprived } \\
\text { vibrissae }\end{array}$ & $\begin{array}{l}\text { Enriched } \\
\text { vibrissae }\end{array}$ & $\begin{array}{l}\text { Deprived } \\
\text { vibrissae }\end{array}$ & \\
\hline ARC B1 C1 D1 (2 rats) & 6 & 34 & 6 & 34 & \\
\hline ROW C1 C2 C3 (2 rats) & 6 & 34 & 6 & 34 & 80 \\
\hline Total vibrassae & \multicolumn{2}{|c|}{80} & \multicolumn{2}{|c|}{80} & 80 \\
\hline
\end{tabular}


subsequently housed in a tactually enriched environment cage or began behavioral training in the texture discrimination task (see below). Rats in the tactually enriched environment stimulation condition were housed together in groups of 6 rats, and maintained in the dark 23 hours daily. Rats in the training group were subjected to binocular occlusion one week before initiating training and housed in separate standard colony cages, except during the daily training session. Binocular occlusion was essential for the elimination of all possible visual cues for problem solving and to assure exclusive vibrissal use in discrimination learning (Guic et al. 92). Binocular occlusion was performed under general anesthesia $(100 \mathrm{mg} / \mathrm{kg}$ intraperitoneal Ketamine) by adhering the superior and inferior palpebral borders in each eye with enbucrilate (Histoacryl).

Apparatus and stimuli. The apparatus and behavioral procedure have been described in detail in earlier reports (Guic et al., 1989; Guic et al., 1992). A jumping box was used to train the subjects to explore tactile discriminative stimuli only by use of vibrissal inputs. The apparatus consisted of a rectangular wooden box $(107 \times 46 \times 50 \mathrm{~cm})$ that had three boxes, one that served as "starting" and two as goal boxes. The rat was given access to a central Y-shaped platform each time that a guillotine door was lifted. This platform was raised $15 \mathrm{~cm}$ off the floor of the box; both divergent arms stood in front of two landing platforms where the discriminative stimuli were presented. The distance between both arms of the $\mathrm{Y}$ and the landing platforms could be adjusted to assure that the rat contacted the rugged surfaces only with its vibrissae. For the same reason, the landing platforms stood $10 \mathrm{~cm}$ higher than the central platform. Stimuli were two sandpaper-lined, U-shaped surfaces that allowed for easy changing at random left-right location of discriminanda. The stimuli covered the front aspects of the landing platforms in a way that allowed rats to explore the tactile stimuli before jumping. The smoother sandpaper had $0.7 \mathrm{~mm}$ mean diameter grains (200 grains $/ \mathrm{cm}^{2}$ ) and the rougher $2 \mathrm{~mm}$ diameter grains (25 grains/ $\mathrm{cm}^{2}$ ). The finer surface was associated with reinforcement (positive stimulus or $\mathrm{E}+$ ) and the rougher with no reinforcement (negative stimulus or E-).

Training in the discrimination task. All rats were initially habituated to the apparatus and were then pre-trained to jump from the central Y-shaped platform onto one of the two landing platforms at an approximate distance of 15 to $20 \mathrm{~cm}$. The distance between the two platforms was gradually increased, moving the Y platform backwards. Rats were required to jump onto one of the landing platforms to obtain food reinforcement. They were allowed to explore the landing platforms with their vibrissae before jumping. However, if the rat explored the landing platform with its nose or paws, no reinforcement was delivered, and it was put again in the starting position on the Y-platform. The optimal jumping distance without paw or nose exploration was empirically determined by observation of the direct and recorded animal behavior. During this early training stage, texture stimuli were not presented to the animal. Positive and negative stimuli were added by the end of the modeling of the requested instrumental behavior, that is, once the rats could easily jump from the central to the landing platforms after exploring the landing platform using only the vibrissae. During discrimination training, each rat received 20 daily trials. A trial started when the experimenter lifted the guillotine door, which allowed the animal access to the $\mathrm{Y}$ platform. A correct response was recorded when the animal jumped onto the landing platform with the $\mathrm{E}+$. In that case, the large guillotine door at the end of the landing platform was opened allowing the animal access to the goal box where two $45 \mathrm{mg}$ food pellets were available as reinforcement. When the subject jumped onto the platform with the E-, it was left on the platform for 5 seconds. Then, a small lateral guillotine door was opened, allowing the animal into the goal box without reinforcement. The right and left positions of the positive and negative stimuli was determined by a random sequence, with the restriction that no stimulus could appear on one side more than three consecutive times. 
Stimuli were removed and reinstalled for each trial, to eliminate auditory cues that might potentially indicate the position of the positive stimulus. Subjects did not have visual cues at their disposal, since they had been binocularly occluded previous to training. Subjects were trained until they reached a criterion of $85 \%$ or more correct responses during 3 consecutive days. In total, rats were trained for approximately 450-500 minutes.

Behavioral monitoring. All trials were directly visualized and videotaped to assure that rats used their long intact vibrissae to solve this sensory discrimination task. This precaution was taken because the skin between the long facial vibrissae has an independent cortical representation. Sensory inputs arising from the skin of the face and vibrissae have been considered parallel and independent sensory routes that process different types of sensory information. A detailed analysis of the video recordings confirmed that exploration of the tactile stimuli by the rats was limited to deflection of the long intact vibrissae. Cut vibrissae, the very short vibrissae on the anterior face and other facial structures were not engaged by the rat in this behavior.

Stimulation in an enriched environment. Rats were put in groups of 6 in a large cage $(80 \times 80 \times 60 \mathrm{~cm})$ containing various objects that tactually enriched the environment, especially objects with different textures (pieces of carpet, cloth, differently rugged stones, etc.) and maintained in the dark. The objects were changed daily to avoid habituation and food location was also changed daily to promote active exploration. In total, rats were exposed to the enriched environment for 4-6 weeks, approximately the same number of days that the trained rats needed to learn the roughness discrimination.

Electrophysiologic recording. By the end of the selective stimulation or behavioral training, the Posteromedial Barrel Sub-field (PMBSF) in SI was mapped in detail using penetrating microelectrodes. Vibrissae were not trimmed for three days prior to recording; instead, the three selectively stimulated vibrissae were cut on the recording day so that their length (approximately $4-5 \mathrm{~mm}$ ) equaled that of the regularly cut ones. The cortical mapping has been detailed elsewhere (Guic et al., 1992; Guic et al., 2008; Jenkins et al., 1990). Briefly, all procedures were done under aseptic conditions, and using sterile electrodes, fluids and instruments. Rats were initially anesthetized with Ketamine (100 $\mathrm{mg} . / \mathrm{kg}$. i.p.), with the femoral vein then canulated to deliver Pentobarbital (dose of $10 \mathrm{mg} . / \mathrm{kg}$, supplemented as needed approximately every 2 hours) and physiological serum during the experiment. A tracheotomy and intubation assured an open airway through the long recording experiment. Rats were placed in a head holder. Rectal temperature was monitored and maintained at $37^{\circ} \mathrm{C}$ using a temperature controlled warming blanket. The condition of the animal was assessed by continuously monitoring EKG and reflexes. A medial incision in the skin of the head and neck and dural perforation at the level of foramen magnum resulted in a continuous drainage of cerebrospinal fluid, to minimize the possibility of cerebral edema. The somatosensory cortex contralateral to the stimulated mystacial pad was exposed by a craniotomy and durotomy, a photograph of the cortical surface vasculature taken, and the cortex maintained under a shallow well filled with liquid silicon. Tungsten microelectrodes (approximately 2 megaohms) were used to record extracellular unit or multiunit potentials from a single depth in the PMBSF in SI. Microelectrodes were introduced approximately normal to the pia surface and advanced through the cortex to a depth of 750 microns from piamater contact, with variations that exceptionally reached a minimum of 650 and a maximum of 850 microns in depth, where robust stimulusdriven responses were most readily recorded. All penetrations in any given animal were parallel to one another. Horizontal positions of the electrode were recorded by reference to details of the cortical vasculature on the image of the cortical surface. In all electrophysiological mapping studies, we made electrode penetrations as close together as the vascular pattern permitted. Receptive fields of neurons were defined using a displacement controlled piezoelectric stimulator, detecting all whiskers whose 100 
microns deflection activated the sampled neuron or cluster producing a detectable response (at least twice the amplitude of the noise signal). Conventional amplification, display and recording methods were used. Neural signals were amplified (differential pre-amplifier Grass P15-C) band-pass filtered (300-5000 Hz) and displayed on an oscilloscope (Tektronik RM 502-A) and audio speaker. We analyzed mostly large amplitude (mv), short-latency extracellular unit or multiunit potentials. In $94.9 \%$ of all recorded responses the latency was shorter than $12 \mathrm{~ms}$ and $70.7 \%$ had a latency between 5 and $8 \mathrm{~ms}$.

Overlapping representational areas were those associated with sites in which we recorded activity from more than one vibrissa, the Principal Vibrissae (PV) being the one that produced the response with the largest amplitude and shortest latency. Adjacent Vibrissae (AV) were the other vibrissae that evoked responses in the same site, almost always neighbors to the PV. Exclusive areas were those in which only the PV evoked a response.

Reconstruction of the PMBS. At the end of the experiment, each unit response sampled was transformed into a polygonal area bounded by dividing lines that were equidistant from the closest sampled sites. These sampled areas, associated with each recording site, were $0.045 \mathrm{~mm}^{2}$ on average, in these studies. The method used allowed for a regular and complete sampling of the evoked units in the PMBSF. Responses were reconstructed from arcs 1-4, and only from rats in which we mapped the entire PMBSF. Using these areas associated with each recording site, the exclusive and nonexclusive (i.e., overlapping) cortical areas of representation were determined.

\section{RESULTS}

\section{Stimulation vs Learning}

As shown in graphs A-D in Figure 1, selectively stimulated vibrissae engaged by active exploration - either in an enriched environment (graphs A and B) or by tactile discrimination (graphs C and D) - presented a significantly larger area of cortical representation than did deprived vibrissae $[\mathrm{F}(3,235)=90.020 ; \mathrm{p}<0.00001$; stimulated vs deprived $(\mathrm{t}=13.873$; d.f. $=142$; $\mathrm{p}<0.00001)$ and trained $\mathrm{vs}$ deprived $(\mathrm{t}=$ 11.549; d.f. $=142 ; \mathrm{p}<=0.00001)]$. No difference was found in this same variable between the type of experience (training vs stimulation: $\mathrm{F}(1,5)=0.307 ; \mathrm{p}<0.803)$ or configuration (row vs arc: $\mathrm{F}(1,5)=0.032$; $\mathrm{p}<0.864$ ), i.e., the effect of selective stimulation was equal for all cases. Figure 2A shows the mean area of total cortical representation of vibrissae in each of the four experimental conditions - normal control, deprived (cut daily), environmentally enriched and trained. Areas of total representation of normal and deprived vibrissae did not differ $(\mathrm{t}=1.477$; d.f. $=210 ; p<0.141)$.

Both, environmental enrichment and training had the common effect of increasing the area of overlap, which was far greater than that recorded for vibrissae in normal control animals, or for deprived vibrissae $\left(X^{2}=61.62 ;\right.$ d.f. $\left.=3 ; p<0.0001\right)$. The extents of overlap did not differ between trained and stimulated vibrissae $(\mathrm{F}(1,5)=0.07 ; \mathrm{p}<0.793)$. However, the areas of exclusive representation of vibrissae trained in roughness discrimination were significantly smaller than were those of vibrissae stimulated in an enriched environment $(\mathrm{F}(1,5)=7.357 ; \mathrm{p}<0.042)$. Thus, learning and stimulation did not affect the areas of exclusive representation in an identical way (see Figure 2c). Further, environmentally enriched vibrissae did not differ from normal controls as to their exclusive areas ( $\mathrm{t}=-0.986 ;$ d.f. $=90$; $\mathrm{p}<0.327)$, while exclusive representational zones for both were larger than for deprived (cut) vibrissae $[\mathrm{F}(2,232)=4.788 ; \mathrm{p}<0.003$; stimulated vs deprived $(\mathrm{t}=-2.633$; d.f. $=142 ; \mathrm{p}<0.009)$, normal vs deprived $(\mathrm{t}=$ 3.127 ; d.f. $=210 ; \mathrm{p}<0.002)]$. On the other hand, trained vibrissae had smaller exclusive CR areas than enriched vibrissae (Mann-Whitney 26.000; p $<0.024 ;$ 2-tailed) and than normally stimulated vibrissae (Mann-Whitney 42.000; $\mathrm{p}<0.021 ; 2$-tailed). Thus, enriched enviroment did not affect the exclusive representational area, while 
learning resulted in its reduction. This result is also seen by comparing areas of exclusive representation between deprived and stimulated vibrissae.

Note that for all variables analyzed thus far (size of total area of representation, exclusive and overlapping areas of cortical representation) there were no differences between the selective row $(\mathrm{C} 1 \mathrm{C} 2 \mathrm{C} 3)$ or arc (B1C1D1) stimulation.

\section{Deprivation}

Areas of exclusive representation. The exclusive area of representation for deprived vibrissae was smaller than for normal vibrissae $(\mathrm{F}=3.127$; d.f. $=210 ; \mathrm{p}<0.002)$, but there was no difference between deprived and normal vibrissae in the extent of representational overlap. Deprivation appears to reduce the area of exclusive representation, without altering the degree of representational overlap (Figure $2 \mathrm{~B}$ and $\mathrm{C}$, for a summary see figure 3 ).

Neither the configuration of stimulation (rows or arcs) nor the type of stimulation (enriched environment or behavioral training) induced any differences in the exclusive cortical representations of deprived vibrissae.
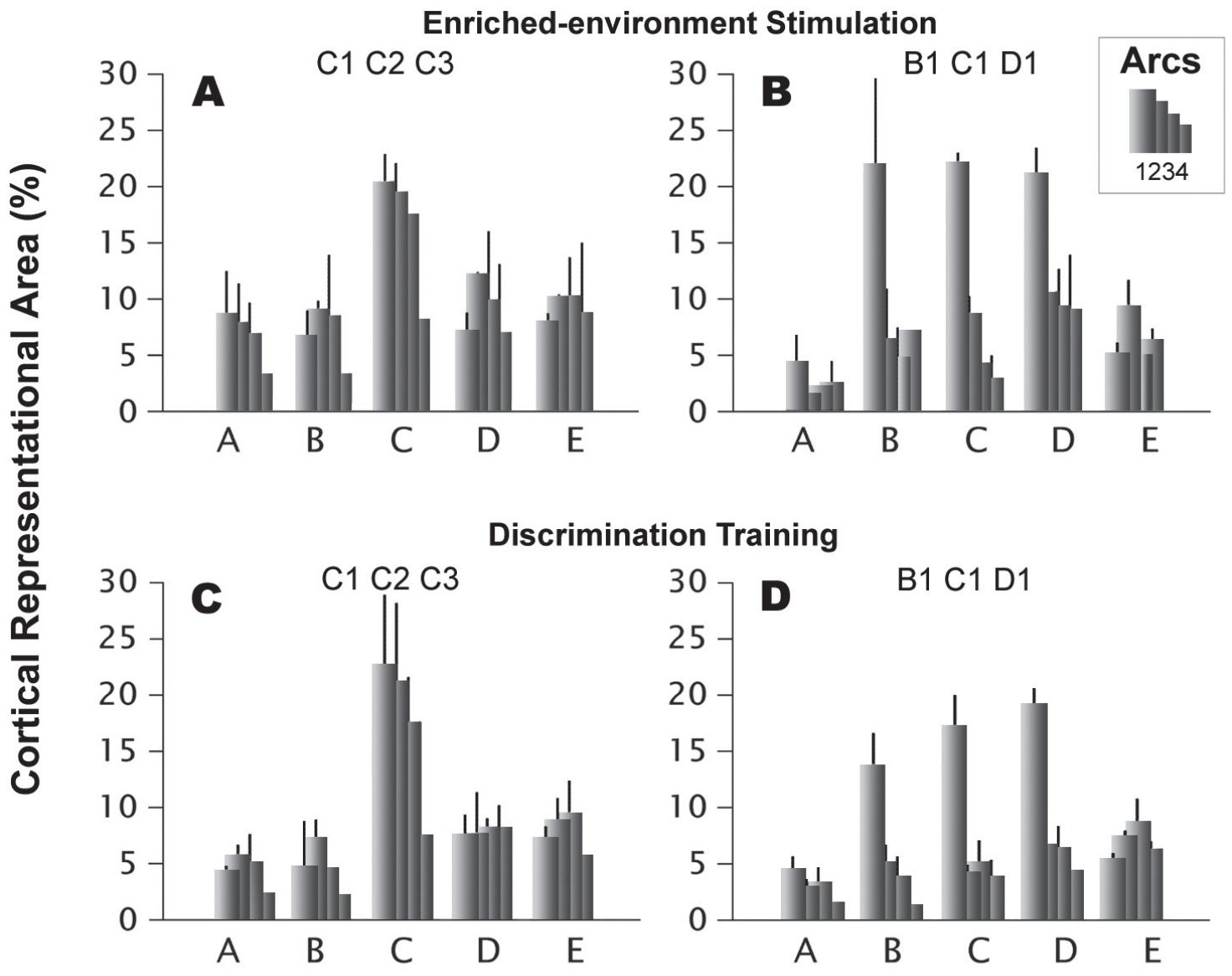

Rows

Figure 1: Average size of cortical representational areas for each vibrissa, as a percentage of the PMBSF. The bars are located resembling the spatial distribution of vibrissae and barrels in arcs and rows. Figures from $\mathrm{A}$ to $\mathrm{D}$ show results for each of the selective stimulation conditions: Tactile enriched environment stimulation for vibrissae C1C2C3 (A) and B1C1D1 (B); Tactile discrimination training for vibrissae $\mathrm{C} 1 \mathrm{C} 2 \mathrm{C} 3$ (C) and $\mathrm{B} 1 \mathrm{C} 1 \mathrm{D} 1$ (D). 
Areas of total cortical representation. The area of total cortical representation of deprived vibrissae was not as homogeneous $(\mathrm{F}(4,127)=8.083 ; \mathrm{p}<0.001)$ as the exclusive areas. The vibrissae in the more dorsal or superior rows presented smaller areas of cortical representation than did those located more ventrally, so that the areas for rows A and B were significantly smaller than for $D$ and $E$, while $C$ was intermediate in size [A vs D ( $t=-4.661 ;$ d.f. $=55$; $\mathrm{p}<0.001) ;$ A vs E $(\mathrm{t}=4.747$; d.f. $=59$; $\mathrm{p}<0.001) ; B$ vs $\mathrm{D}(\mathrm{t}=-3.191 ; \mathrm{d} . \mathrm{f} .=53$; $\mathrm{p}<0.002) ; B$ vs $\mathrm{E}(\mathrm{t}=-3.006 ; \mathrm{d} . \mathrm{f} .=57$; $\mathrm{p}<0.004) ; \mathrm{C}$ vs $\mathrm{D}(\mathrm{t}=-2.452 ;$ d.f. $=42 ; \mathrm{p}<$ $0.018)]$. The rows constituted different sets, formed by relatively homogeneous elements, so that if the whole was considered from the perspective of the arcs, they did not differ from one another. The sizes of the areas of overlap tended to follow the same pattern as did those describing the areas of total cortical representation.

Figure 3 presents a graphic summary of the results presented thus far. It shows that deprivation and training reduced the areas of exclusive representation, while both selective stimulation procedures increased the areas of overlap. The sizes of total cortical representation of stimulated vibrissae were due mainly to an increase in the area of the overlap, rather than to an increase in the size of the areas of exclusive vibrissal representation.

\section{Directional bias of the overlapping areas}

As seen in section I, the increase in cortical representation was mainly reflected by the growth of overlapping representational territories. The configuration of arc (B1C1D1) or row (C1C2C3) stimulation can drastically alter the proportion of cortical area that one vibrissa shares with others in its same row or arc $(F(1,5)=$ 49.244; $\mathrm{p}<0.001)$. The same effect was recorded for tactually enriched and tactually trained rats $(\mathrm{F}(1,5)=1.485$; $\mathrm{p}<0.277)$. Since there were no differences in the direction of overlap between vibrissae stimulated in an enriched environment and those subjected to learning in a tactile task, we shall use the name "selective stimulation" when referring to either situation.

It was interesting to verify that even if there were differences in the extents of overlap, the proportions in which vibrissae shared cortical responses with their row and arc neighbors were the same for vibrissae selectively stimulated in a row as they were for normal and deprived vibrissae (Figure

\section{Cortical Representational Areas}
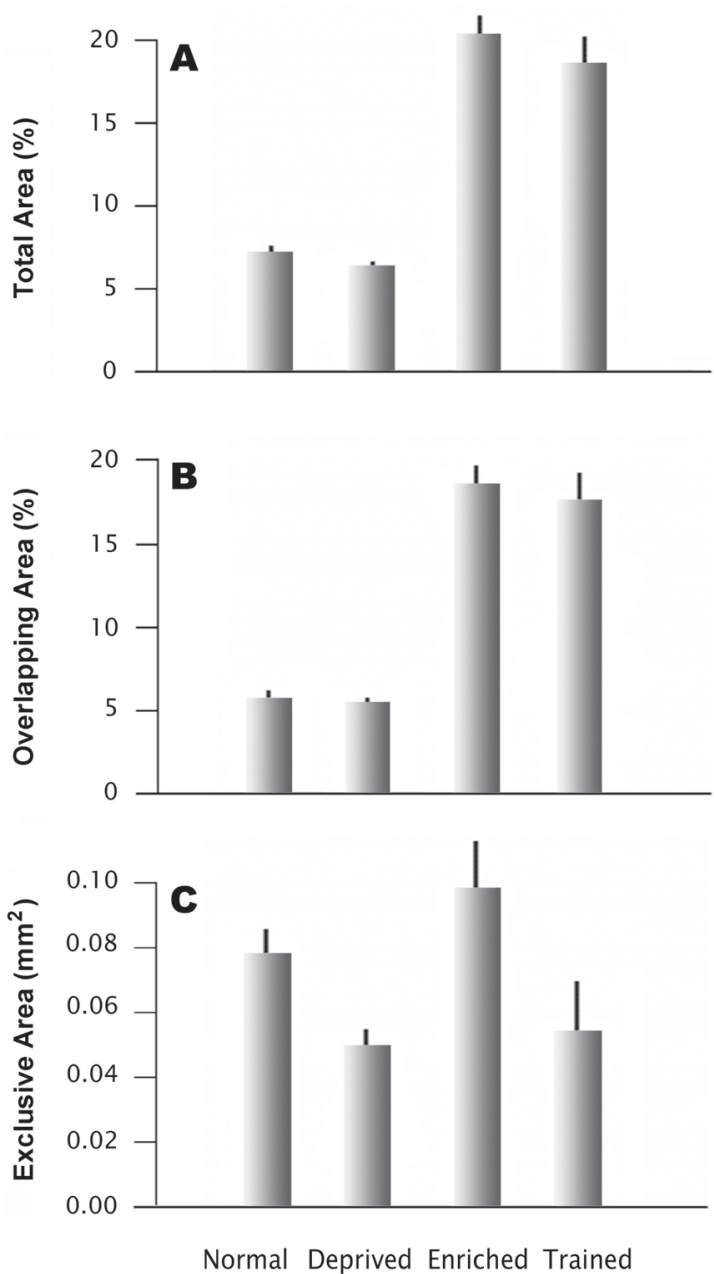

Figure 2: Size of cortical representational areas, as a percentage of the PMBSF. Bars show results for vibrissae in each of the experimental conditions. Total representational areas (A); Overlapping zones (B) and exclusive cortical areas $(\mathrm{C})$. 
4). Thus, although the total area of representation of trained or enriched vibrissae grew several-fold, still, as for normal and deprived vibrissae, approximately $70 \%$ of the total area of overlap responded to the stimulation of other vibrissae in the same row and about $30 \%$ responded to other vibrissae in the same arc. This difference was equivalently significant in all groups [Normal vibrissae: $\mathrm{t}=11.77, \mathrm{gl}=234 ; \mathrm{p}<0.0001$. Row stimulated: $\mathrm{t}=8.026, \mathrm{gl}=11, \mathrm{p}<0.0001$. Deprived: $\mathrm{t}=9.221, \mathrm{gl}=130 ; \mathrm{p}<0.0001]$.

However, whenever the three stimulated vibrissae belonged to the same arc, the proportion was reversed, and the vibrissae shared approximately $70 \%$ of their area of overlap with other vibrissae in their arc and only $30 \%$ of the overlap with others in the same row $(t=5.92, \mathrm{gl}=11, \mathrm{p}<0.0001)$.

These results suggest that vibrissae stimulated in a row $(\mathrm{C} 1 \mathrm{C} 2 \mathrm{C} 3)$ increased their area of cortical representation by the enlargement of the area of overlap between them but also with their neighbors along the arc. On the other hand, the growth of areas of representation of vibrissae stimulated in an arc (B1C1D1) was due to enlargement of the domain of overlap only between them. These results are signaled by arrows in Figure 4.

This is consistent with the direction of overlap of deprived vibrissae representations bordering (adjacent to) environmentally or behaviorally stimulated vibrissae. Vibrissae B2 and D2 are deprived vibrissae adjacent to the stimulated vibrissae in the case in which B1C1D1, as well as when $\mathrm{C} 1 \mathrm{C} 2 \mathrm{C} 3$, were stimulated. B2 and D2 are neighboring vibrissae in the same row to the ones stimulated in arc (B2D2-arc) and are neighbors in the same arc to the ones stimulated in row (B2D2row). The size of the total area of overlap was equal for both groups of B2 and D2 $(\mathrm{F}(1,6)=0.241, \mathrm{p}<0.644)$ but vibrissae B2D2-row have the largest percentage of overlap in the direction of the arc, as compared to vibrissae B2D2-arc $(\mathrm{t}=2.313$; d.f. $=14 ; \mathrm{p}<0.05)$, to "non-adjacent" deprived ones $(t=02.427$; d.f. $=89 ; \mathrm{p}<0.017)$ and to normal ones. This is consistent with the fact that $\mathrm{CR}$ areas of vibrissae stimulated in a row $(\mathrm{C} 1 \mathrm{C} 2 \mathrm{C} 3)$ also grow toward the arc, not only share larger cortical areas amongst them.

\section{Cortical Representational Areas in Different Stimulation Conditions}

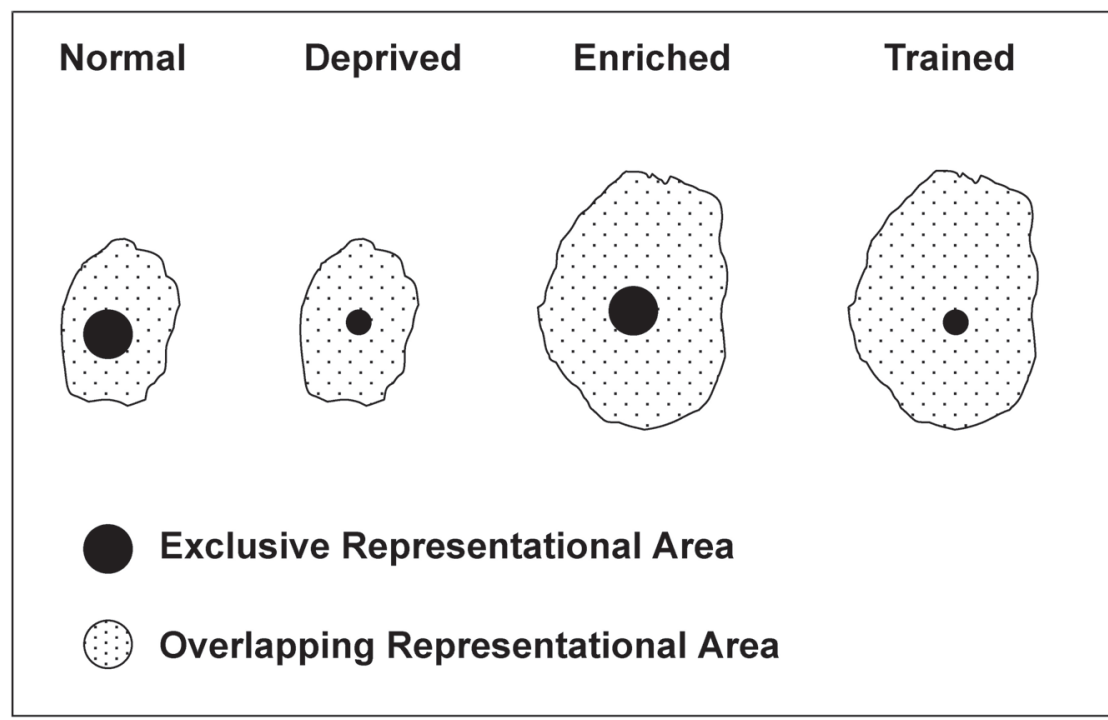

Figure 3: Size of the cortical representation areas in different experimental conditions. The black central circles represent the sizes of exclusive areas; dotted sectors represent the sizes of the overlapping representational zones for the principal vibrissae. See text for explanation. 


\section{Directional Bias of Representational Overlapping}
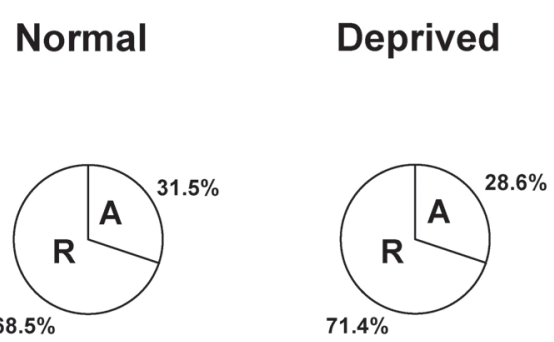
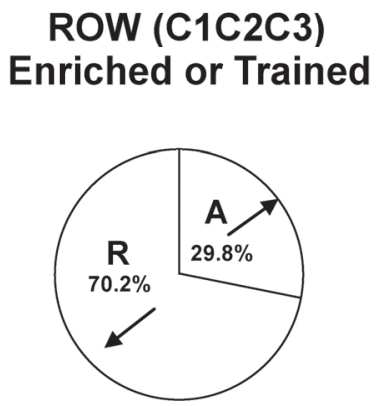

\author{
ARC (B1C1D1) \\ Enriched or Trained
}

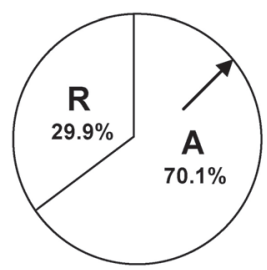

Figure 4: Directional bias of representational overlap. The size of each circle represents the total size of the zone of representational overlap, for each experimental condition. In each circle, the directional bias of overlap of CR towards the same Arc (A) and Row (R) is shown. Overlapping areas for normally stimulated vibrissae, deprived vibrissae and for selectively stimulated vibrissae in a row $(\mathrm{C} 1 \mathrm{C} 2 \mathrm{C} 3)$ or in an arc $(\mathrm{B} 1 \mathrm{C} 1 \mathrm{D} 1)$, are shown. Arrows signal enlargement of overlapping cortical areas along arcs and rows in selectively stimulated (enriched or trained) compared to normally stimulated or deprived vibrissae.

\section{DISCUSSION}

\section{Effects of environmentally enriched} stimulation and discrimination learning. The selective stimulation of 3 vibrissae in a tactile enriched environment produced a 3to-4-fold increase in the cortical areas of their representation in SI. These positive changes were far greater than those recorded in earlier barrel field plasticity experiments (Fox et al., 2000; Diamond et al., 1993; Polley et al., 1999)

For environmentally stimulated rats, it is hypothesized that recorded differences are due to a more rigorous control of the conditions of environmental enrichment, achieved by daily changing cage environment and food source locations, thus making sure that the rats actively explored tactile cues to operate in their environment, and also by extending the environment enrichment period to 4-6 weeks.

Our results confirm that behaviorally relevant stimulation was an important factor generating a large increase in cortical representation. Stimulation per se is inadequate. When appropriate conditions of behavioral context are not met, no change, or even negative changes, may be the result
(Welker et al., 1992; Polley, 2004). These findings substantially differ from the changes produced by behaviorally relevant stimulation versus deprivation in our study (See Figure 3). Behaviorally relevant stimulation in continuously enriched environments resulted in an increased area of overlap of the cortical representation of engaged vibrissae. Areas of exclusive cortical representation of vibrissae were not altered. By contrast, deprivation achieved by daily trimming of whiskers in the same rats did not affect the area of overlap, but it did result in a decrease in the area of exclusive SI cortical representation.

The engagement of vibrissae in the context of a daily 20-minute long discrimination task resulted in a dramatic increase in the area of shared representation, and in a decrease in the area of exclusive representation of the engaged vibrissae. Note again, that these changes were produced by an average of 480 discriminative training trials that amounted to a total average period of only 480 minutes of behavioral training. That is in contradistinction to environmentally enriched rats, which operated continuously in enriched novel environments for a period of 4-6 weeks. 
In general, learning-driven increases in cortical representation have been attributed to coincident input-dependent learning processes (Merzenich, 2001; Merzenich \& Sameshima, 1993; Weinberger, 1995). Those processes would be expected to contribute both to the enlargement of overlap of the representations of engaged vibrissae in both experimental groups, and to the change in the direction of the overlapped areas. The smaller area of exclusive vibrissae representation in trained vs enriched rats might hypothetically arise from a more consistent and stereotypic schedule of whisker co-stimulation in roughness discrimination than in exploring a variety of new surfaces and objects in a continuously changing (enriched) environment.

These results are in striking contradistinction to studies by Polley et al. (2004), who recorded significant reductions in electrophysiologically recorded receptive field sizes and in the areas of cortex activated during optical imaging by stimulation of any single vibrissa, in animals that were maintained in an enriched environment with all vibrissae intact. Differences in outcomes strongly support the argument that the changes in CR that we report here were a product of competitive Hebbian network plasticity (Hebb, 1949). By that interpretation, the dramatic enlargement of the representations of intact, behaviorally-engaged vibrissae is attributable to their competitive dominance over the inactive, surrounding short vibrissae. In the study of Polley et al. (2004), vibrissae also compete for territorial domination, but with all competitors intact, the effect of behavioral engagement is the progressive refinement (not enlargement) of selective cortical representations.

At the same time, these contrasting studies raise a provocative question about the nature of the observed plastic changes: Why is competition between the three long vibrissae arrayed in arcs or rows in the current study not more pronounced? In our experiment, those vibrissae were very strongly overlapped with one another, over very long cortical distances. Further studies will be required to understand why stronger competitive effects were not recorded between the three long, heavilybehaviorally-engaged whiskers in our experiments.

It might be noted that similar observations were recorded earlier by Recanzone et al. (1992) in the somatosensory cortex of adult monkeys. They showed that the receptive fields and cortical territories of representation underwent a several to many fold enlargement in a monkey performing a temporal frequency difference discrimination task, under the condition that all training applied to a fixed, small, unchanging spot on the middle segment of one finger. That skin location could be regarded as a competitive winner in a competitive Hebbian network. After learning, its representation grew dramatically, as did the average receptive field size. On the other hand, when the skin location was changed every day to different sites on the same digit in a monkey performing the same behavioral task, exactly the opposite consequences were recorded: The areas of representation of any given skin site were reduced from normal, as was the average receptive field size. As in Polley's study, each site on the sensory input array hypothetically competed for cortical response domination. In this active competitive environment, the skin surface came to be represented in significantly finer detail. In our study, as in the initial fixedlocation monkey behavior, given vibrissae and the fixed skin location on the monkey's finger-quickly grew because of its strong competitive advantage.

Relation between cortical columns. The relation between cortical columns was studied by analyzing the extents of overlapping cortical areas that a vibrissa shared with its neighbors in the same (or different) row or arc. As a result of plastic changes, neuronal activity was evoked by stimulation in interbarrel zones, as well as all across the zones of representation of neighboring vibrissae. It should be remembered that this unit response sample was from cortical layer 4 and may reflect significantly altered thalamic input strength. 
If the dramatic increase of the area of overlap was due to the co-activation of three vibrissae, as was earlier hypothetized, it is interesting to note that changes were equivalently dramatic if the 3 vibrissae were arrayed in either a row or an arc. One might anticipate that nearly simultaneous stimulation of vibrissae could be stronger for an arc than for a row triad. Whether that was the case or not, the dramatically growing overlap was strongly biased to involve the other two long stimulated vibrissae, regardless of whether they were arranged in an arc or a row (also see, Diamond et al., 1993; Armstrong-James et al., 1994; Lebedey et al., 2000).

Note that when stimulating $\mathrm{C} 1 \mathrm{C} 2 \mathrm{C} 3$ i.e. vibrissae in the same row - the sizes of the total areas of overlap increased, while the same proportion between the areas that the vibrissa shared with others in its row and in its arc were maintained. By contrast, when B1C1D1 were stimulated together i.e. vibrissae in the same arc - the area of overlap again increased to approximately match the overlap recorded for the row trial stimulation, but in this case, the normal proportionately biased to stronger row vs arc overlap was reversed. This effect is consistent with the interpretation that Hebbian plasticity underlies these largescale representational changes.

\section{ACKNOWLEDGMENTS}

We dedicate this work to our friend and mentor Dra. Teresa Pinto-Hamuy who enthusiastically supported this research. DIUC \#91038 and FONDECYT \#1930721 to E. Guic.

\section{REFERENCES}

ARMSTRONG-JAMES M, DIAMOND M, EBNER FF (1994) An innocuous bias in whisker use in adult rats modifies receptive fields of barrel cortex neurons. The journal of Neuroscience 14(11): 6978-6991

BRECHT M (2007) Barrel cortex and whisker-mediated behaviors. Current Opinion in Neurobiology 17: 408416

CALFORD MB, TWEEDALE R (1991) Acute changes in cutaneous receptive fields in primary somatosensory cortex after digit denervafion in adult flying fox. Journal of Neurophysiology, 65(2): 178-187

DIAMOND ME, ARMSTRONG-JAMES M, EBNER FF (1993) Experience-dependent plasticity in adult rat barrel cortex. Proceedings of the National Academy of Sciences USA 90: 2082-2086

FOX K, GLAZEWSKI S SCHULZE S (2000) Plasticity and stability of somatosensory maps in the thalamus and cortex. Current Opinion in Neurobiology 10: : 494497

GLAZEWSKI S, FOX K (1996) The time course of experience-dependent synaptic potentiation and depression in barrel cortex od adolescent rats. Journal of Neurophysiology 75: 1714-1729

GUIC-ROBLES E, VALDIVIESO C, GUAJARDO G (1989) Rats can learn a roughness discrimination using only their vibrissal system. Behavioural Brain Research 31: $285-289$

GUIC-ROBLES E, JENKINS W, BRAVO H (1992) Vibrissal roughness discrimination is barrel-cortex dependent. Behavioural Brain Research 48: 145-152

GUIC E, CARRASCO X, RODRÍGUEZ E, ROBLES I, MERZENICH M (2008) Non-homogeneous spatial configuration of vibrissae cortical representation in layer IV of the barrel somatosensory cortex. (Submitted to Biological Research)

HEBB DO, (1949) The organization of behavior: a neuropsychological theory. New York: Wiley.

JENKINS WM, MERZENICH MM, OCHS MT, ALLARD T, GUIC-ROBLES E (1990) Functional reorganization of primary somatosensorv cortex in adult owl monkeys after behavioral controlled tactile stimulation. Journal of Neurophysiology 63 (1): 82-104

KAAS JH, KUBITZER LA, CHINO YM, LANGSTON AL, POLLEY EH, BLAIR N (1990) Reorganization of retinotopic cortical maps in adult mammals after lesions of the retina. Science 248: 229-231

KAAS JH (1991) Plasticity of sensory and motor maps in adult mammals. Annual Review of Neuroscience 14: 137-167

KALASKA J, POMERANZ B (1979) Chronic paw deafferentafion causes an age-dependent appearance of novel responses from forearm in "paw cortex" of kittens and adult cats. Journal of Neurophysiology 42: 618-633

KRUPA DJ, GHAZANFAR AA, NICOLELIS MA (1999) Immediate thalamic sensory plasticity depends on corticothalamic feedback. Proceedings of the National Academy of Sciences USA 96: 8200-8205

LEBEDEY MA, MIRABELLA G, ERCHOVA I, DIAMOND ME (2000) Experience-dependent plasticity of the rat barrel cortex: redistribution of activity across barrel-columns. Cerebral Cortex 10: 10231

MERZENICH MM, KAAS J.H, WALL JT, SUR M, NELSON RJ, FEILEMAN DJ (1983a) Topographic reorganization of somatosensory cortical areas $3 \mathrm{~b}$ and 1 in adult monkeys following restricted deafferentation. Neuroscience 8: 33-55

MERZENICH MM, KAAS JH, WALL JT, SUR M, NELSON RJ, FELLEMAN DJ (1983b) Progression of change following median nerve section in the cortical representation of the hand in areas $3 b$ and 1 in adult owl squirrel monkeys. Neuroscience 10: 639-665

MERZENICH MM, NELSON RJ, SRYKER MP, CYNADER MS, SCHOPPMANN A, ZOOK JM (1984) Somatosensory cortical map changes following digit amputation in adult monkeys. Journal of Comparative Neurology, 224: 591-605

MERZENICH M, SAMESHIMA K (1993) Cortical 
plasticity and memory. Current Opinion in Neurobiology 3: 187-196

MERZENICH, M; WRIGHT B; JENKINS W; XERRI C, BYL N; MILLER S AND TALLAL P (2001) Cortical Plasticity underlying perceptual, motor and cognitive skill development: Implications for neurorehabilitation. In: Brain Development and Cognition, Johnson $\mathrm{MH}$; Munakata $\mathrm{Y}$ and Gilmore RO (Eds). Blackell Publishing

PARKER JL, DOSTROVSKY JO (1999) Cortical involvement in the induction but not expression of thalamic plasticity. Journal of Neuroscience 19: 86238629

PETERSEN C (2007) The functional organization of barrel cortex. Neuron 56: 339-355

POLLEY DB, CHEN-BEE CH, FROSTIG RD (1999) Two directions of plasticity in the sensory deprived adult cortex. Neuron 24: 623-637

POLLEY D, KVASNAK E, FROSTIG R (2004) Naturalistic experience transforms sensory maps in the adult cortex of caged animals. Nature 429: 67-71

PONS TP, GARRAGHTY PE, MISHKIN M (1988) Lesioninduced plasticity in the second somatosensory cortex of adult macaques. Proceedings of the National Academy of Sciences USA 85: 5279-5281

RASMUSSON, D (1982) Reorganization of raccoon somatosensory cortex following removal of the fifth digit. Journal of Comparative Neurology 205: 313-326
RASMUSSON DD, DYKES RW (1988) Long-term enhancement of evoked potentials in cat somatosensory cortex produced by co-activation of the basal forebrain and cutaneous receptors. Experimental Brain Research 70: $276-286$

RECANZONE GH, LERZENICH MM, JENKINS WM (1992) Frequency discrimination training engaging a restricted skin surface results in an emergence of a cutaneous response zone in cortical area 3a. Journal of Neurophvsiology 67(5): 1057-1070

WALL JT, CUSICK CG (1984) Cutaneous responsiveness in primary somatosensort (si) hindpaw cortex before and after partial hindpaw deafferentation in adult rats. The Journal of Neuroscience 4: 1499-1515

WALLACE H, FOX K (1999) Local cortical interaction determines the form of cortical plasticity. Journal of Neurobiology 41: 58-63

WEINBERGER NM (1995) Dynamic regulation of receptive fields and maps in the adult sensory cortex. Annual Review on Neuroscience 18: 129-158

WELKER E, RAO SB, DORFL J, MELZER P, VANDERLOOS H (1992) Plasticity in the barrel cortex of the adult mouse: effects of chronic stimulation upon deoxyglucose uptake in behaving animal. The Journal of Neuroscience 12(1): 153-170

XU J, WALL JT (1999) Evidence for brainstem and suprabrainstem contributions to rapid cortical plasticity in adult monkeys. Journal of Neuroscience 19: 7578-7590 